\title{
Low-temperature scanning tunneling microscopy of ring-like surface electronic structures around Co islands on InAs(110) surfaces
}

\author{
D. A. Muzychenko, S. V. Savinov, N. S. Maslova, V. I. Panov \\ Faculty of Physics, Moscow State University, 119992 Moscow, Russia \\ K. Schouteden, C. Van Haesendonck \\ Laboratory of Solid-State Physics and Magnetism, BE-3001 Leuven, Belgium
}

(Dated: October 28, 2018)

\begin{abstract}
We report on the experimental observation by scanning tunneling microscopy at low temperature of ring-like features that appear around Co metal clusters deposited on a clean (110) oriented surface of cleaved p-type InAs crystals. These features are visible in spectroscopic images within a certain range of negative tunneling bias voltages due to the presence of a negative differential conductance in the current-voltage dependence. A theoretical model is introduced, which takes into account non-equilibrium effects in the small tunneling junction area. In the framework of this model the appearance of the ring-like features is explained in terms of interference effects between electrons tunneling directly and indirectly (via a Co island) between the tip and the InAs surface.

PACS numbers: 68.35.Dv, 68.37.Ef, 73.20.At
\end{abstract}

\section{INTRODUCTION}

After the pioneering work of Tsui [1], two-dimensional (2D) electron systems have been intensively investigated, in particular because of their huge application potential for the manufacturing of field effect transistors. Since then, a lot of success has been obtained with respect to improving the stability and the carrier mobility of the $2 \mathrm{D}$ electron gas. On the other hand, the $2 \mathrm{D}$ electron gas provides an excellent playing ground for studying the physical properties of low-dimensional systems. Up to now, the vast majority of experiments have been performed relying on tunneling contacts with planar geometry, where data is inevitably averaged and no local (on nanometer scale) information can be obtained. The nanometer size tunneling contact, which is created in scanning tunneling microscopy (STM) and scanning tunneling spectroscopy (STS) experiments, is able to directly provide such local information about the $2 \mathrm{D}$ electron gas.

Here, we present the results of local measurements of the electronic properties of a 2D system, which consists of metal Co islands deposited in situ on the (110) oriented surface of InAs that is obtained by cleavage in ultra-high vacuum (UHV) of a single crystal. It is well known that by deposition of only a small amount of different adsorbates onto the InAs(110) surface, the Fermi level can be shifted into the conduction band by up to $600 \mathrm{meV}$ (see, e.g., [2, 3, 4] and references cited therein), thus causing strong downward band bending and creating a 2D surface electron channel.

\section{EXPERIMENT AND RESULTS}

\section{A. Experiment}

We performed combined STM and STS measurements with a commercially available low-temperature STM [5]. The STM operates under UHV conditions at a base pressure in the $10^{-12}$ mbar range and at temperatures down to $4.5 \mathrm{~K}$. In order to improve measurement stability, the whole UHV setup is decoupled from the building by a specially designed vibration isolation floor. Electrochemically preformed tungsten tips [5], which are flash annealed in situ at elevated temperatures, are used. The tip quality is routinely checked by acquiring atomicresolution images for the "herringbone" reconstruction of the $\mathrm{Au}(111)$ surface.

Spectroscopic measurements were performed in the current imaging tunneling spectroscopy (CITS) mode [6] with a grid size up to $200 \times 200$ points. Additionally, we relied on harmonic detection with lock-in amplification for "hardware" measurements of the differential conductance. The excitation frequency was in the range $300-7000 \mathrm{~Hz}$, while the excitation amplitude was in the range $5-100 \mathrm{mV}$.

The investigated InAs samples are doped with Mn at a doping level of $5 \times 10^{17} \mathrm{~cm}^{-3}$. At this doping level $\mathrm{Mn}$ in InAs is expected to act as a shallow acceptor with ionization energy around $28 \mathrm{meV}$. InAs slabs with size $5 \times 2 \times 2 \mathrm{~mm}^{3}$ were cleaved in-situ at room temperature in the UHV preparation chamber (base pressure is about $5 \times 10^{-11}$ mbar) that is attached to the UHV STM chamber. Co islands were deposited by means of electronbeam evaporation at a rate of $0.007 \pm 0.001$ monolayer (ML) per second. The evaporation rate was calibrated by determining with x-ray reflectometry the thickness of thick Co films. As discussed in more detail below, the evaporation rate inferred from STM images of the Co is- 


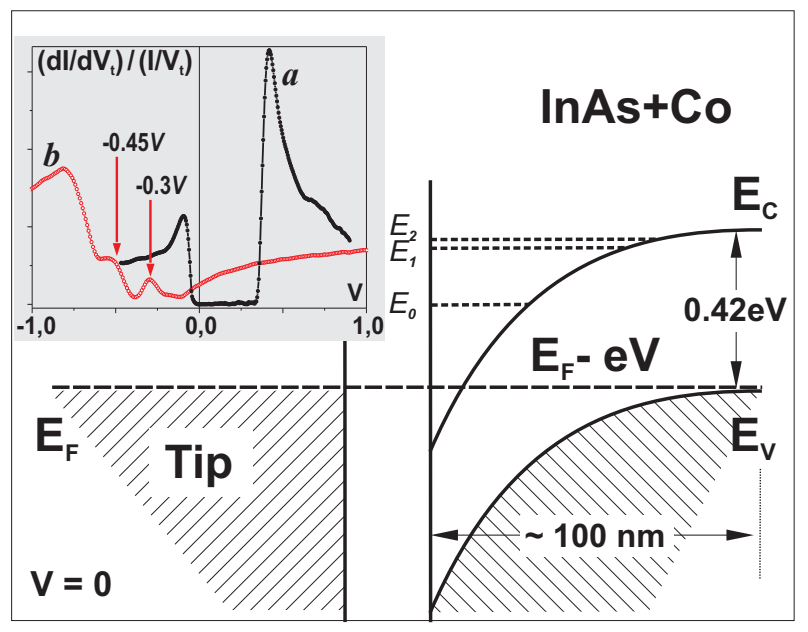

FIG. 1: Schematic diagram of the surface band bending at the p-type InAs(110) surface after Co metal cluster deposition. The band gap value, the width of the depletion layer and the position of the 2D subbands are shown. Experimental normalized tunneling conductance curves are shown in the inset both for the original, clean p-type InAs(110) substrate and for the n-type Co covered substrate (Co-InAs system). Color online.

lands on the InAs surface can deviate considerably from the calibrated rate.

For the $\operatorname{InAs}(110)$ surface, filled and empty surface bands are located deep inside the conduction and valence bands, respectively, and are separated by a $1.7 \mathrm{eV}$ surface gap [7, 8] for a clean surface. Under clean conditions the bands are either flat, or a weak accumulation layer is formed on the surface [9]. However, surface states can be strongly affected within 2 to 3 hours when the base pressure exceeds $(2-3) \times 10^{-10}$ mbar [10]. In order to prevent any undesirable effects of adsorbate induced surface degradation, we always keep the pressure below $10^{-10}$ mbar in the preparation chamber of our UHV setup during the different sample manipulations.

All the reported STM/STS experiments are performed at liquid helium temperature $(\simeq 4.5 \mathrm{~K})$. Everywhere in the text the tunneling bias voltage $V_{t}$ refers to the sample voltage, while the STM tip is virtually grounded. The experiments consist of three stages. First, the clean surface of a cleaved p-type Mn doped InAs(110) single crystal is characterized in detail by STM/STS measurements. Second, Co metal atoms are deposited on the $\operatorname{InAs}(110)$ substrate that is still cold (well below room temperature) after removal from the STM chamber. The amount of Co deposited on the surface is about 0.02 ML. Deposition of a monolayer is according to the definition given in [3]. Third, the Co-InAs system is investigated by STM/STS measurements.

\section{B. 2D surface of Co-InAs}

The quality of the clean cleaved InAs(110) surface is first carefully checked. Stable STM images of the surface reveal (1x1) relaxation with typical "butterfly" type of patterns appearing at the Mn doping atom sites [11], superimposed upon the InAs atomic lattice (see Fig. 2(a)). $\mathrm{Mn}$ atoms can be visualized in the first few subsurface layers. Our STS measurements clearly confirm the ptype character of the surface conductivity (see inset in Fig. 1. curve a), as expected for Mn doped InAs. After deposition of a very small amount of Co atoms (only $0.02 \mathrm{ML}$ ) onto the surface, the general outlook of the normalized $\left(d I / d V_{t}\right) /\left(I / V_{t}\right)$ tunneling conductance curves drastically changes (see inset in Fig. 1. curve b). The Fermi level $E_{F}$ is now positioned about $0.12 \mathrm{eV}$ above the conduction band bottom. Two distinct peaks are visible on the normalized tunneling conductance curves around $-0.30 \mathrm{eV}$ and around $-0.45 \mathrm{eV}$.

In order to provide a consistent explanation for the observed tunneling conductance curves, we need to take into account surface band bending (see Fig. 1). Co clusters are acting as "donors" when grown on a clean InAs(110) surface [3]. The Co clusters are positively charged and downward band bending occurs at the surface. We note that the average value of the positive charge residing on a cluster is not an integer multiple of a single electron charge [12].

Since our results indicate that $E_{F}-E_{C} \simeq 0.12 \mathrm{eV}$, with $E_{C}$ the conduction band edge, and keeping in mind that the band gap for InAs is $0.42 \mathrm{eV}$ at $4.5 \mathrm{~K}$ [13], we then obtain a band bending at the surface that is as large as $0.54 \mathrm{eV}$. This gives rise to a deep quantum well with a few discrete eigenstates. The binding energy of the lowest eigenstate (obtained by solving the PoissonSchrödinger equation following [2]) is $E_{0} \simeq 0.24 \mathrm{eV}$. Consequently, the eigenstate is located almost $0.30 \mathrm{eV}$ above $E_{C}$, or $0.18 \mathrm{eV}$ above $E_{F}$. To make this state visible in the tunneling conductance curve, it has to be shifted inside the tunneling window $\left[E_{F}, E_{F}-e V_{t}\right]$, and this can be achieved by making the tunneling bias voltage sufficiently negative. When the eigenstate $E_{0}$ reaches $E_{F}$, a peak will appear in the normalized tunneling conductance curve. We assume that this happens at a sample bias voltage of about $-0.30 \mathrm{~V}$ for our experiments. The next peak appearing in the normalized tunneling conductance curve at $V_{t} \simeq-0.45 \mathrm{~V}$ can be assigned in a similar way to the fact that the eigenstate $E_{1}$ falls below $E_{F}$. This is similar to what was described for the $\operatorname{GaAs}(110)$ surface in [14].

The top of valence band, $E_{V}$, is located $0.54 \mathrm{eV}$ below the Fermi level. Consequently, at bias voltage $V_{t}=$ $-0.45 \mathrm{eV}$ the width of the depletion layer is relatively small, and a tunneling current is able to flow from the InAs valence band to the tip. This can explain why the peak at $V_{t} \simeq-0.45 \mathrm{~V}$ is superimposed on a wide shoulder that results from the bulk valence band states (see inset in Fig. 1, curve b) 


\section{Rings around Co clusters}

According to the results of our STM measurements, Co atoms tend to form small clusters on the InAs(110) surface despite the low substrate temperature during deposition. A typical STM image of a Co-InAs surface is presented in Fig. 2(a). From Fig. 2(a) we infer that the average cluster size is around $1.5 \mathrm{~nm}$ for a tunneling bias voltage of $-0.65 \mathrm{~V}$. It is important to explicitly refer to the value of the tunneling bias voltage when analyzing topographical STM images, because the apparent size of the Co clusters strongly depends on the applied bias voltage. The majority of the clusters are one monolayer in height. A rough estimate gives an average number of Co atoms in a cluster (assuming a Co atom diameter of about $0.25 \mathrm{~nm}$ ) that is around 30 atoms. There are 21 Co clusters on the STM image in Fig. 2(a), which has a size of $4.7 \times 4.7 \mathrm{~nm}^{2}$. Consequently, the coverage that is inferred from Fig. 2(a) is $0.068 \mathrm{ML}$, i.e., 3 to 4 times larger than the coverage obtained from the calibrated deposition rate.

A very intriguing observation in our STM images of the Co islands on the InAs(110) surface is the observation of ring-like features that appear around the Co clusters. In Fig. 2(a) one observes more or less circular depressions with sharp edges around some of the Co clusters. A surprising fact about these depressions is that the depressions have a different diameter at the same value of the bias voltage for clusters that look very similar in the STM image. In previous STM experiments [15], similar features were observed around metal clusters of unknown origin.

In order to obtain a more detailed insight into the appearance of the ring-like features, we performed spatially resolved spectroscopic measurements above the same surface area where the STM image was obtained (Fig. 2(a)). In a first set of experiments, we relied on imaging based on harmonic detection with a lock-in amplifier. The results are shown in Figs. 2(b), (c), (d) and (f). Each image corresponds to a map of the tunneling current response at the frequency that is used for the modulation of the tunneling bias voltage. In a first approximation, this response is proportional to the value of the local density of states (LDOS) $\rho$ according to the relation

$$
d I / d V_{t}\left(x, y, V_{t}\right) \propto \rho\left(x, y, E-E_{F}=e V_{t}\right) .
$$

Next, we performed high-resolution CITS measurements with grid size of $130 \times 130$ points. Figure $2(\mathrm{e})$ shows a set of $I\left(V_{t}\right)$ curves acquired in the CITS mode along the cross-section $a a^{\prime}$ indicated in Fig. 2(c). Regions with negative slope, which corresponds to a negative differential conductance (NDC), can be clearly observed on all but the two lowest curves. The latter two curves were acquired near point $a$, i.e., at points that are at the largest distance from the cluster. The closer to the cluster the $I\left(V_{t}\right)$ dependence is measured, the lower in absolute value is the tunneling voltage at which the region with NDC occurs. This experimental finding remains intact for STM tip to cluster distances down to a few tenths of $1 \mathrm{~nm}$. Comparing Fig. 2(e) with Figs. 2(b), (c), (d) and (f), we come to the conclusion that the distance $d$ between the STM tip and the cluster determines the bias voltage $V_{N D C}(d)$ around which the NDC occurs on the $I\left(V_{t}\right)$ curve. A dark ring with radius $d$, which can be directly related to the presence of the NDC occurring around $V_{N D C}(d)$, will appear on the $d I / d V_{t}\left(x, y, V_{t}\right)$ image taken near the bias voltage $V_{N D C}(d)$.

From our analysis of the results shown in Fig. 2 we conclude that (i) sharply defined dark rings appear in the differential conductance (LDOS) images around some of the clusters within a certain range of negative tunneling bias voltages, (ii) the size of the dark rings is shrinking when the absolute value of the tunneling bias voltage is decreased, and (iii) the LDOS has an almost constant value above the Co-InAs surface except for the clusters and the immediate vicinity of the dark rings. The only image, which reveals pronounced spatial variations of the normalized tunneling conductance, is image Fig. 2(c), taken at the threshold value of the tunneling bias voltage $V_{t M A X}$ that approximately corresponds to the bias voltage where the ring radius reaches a maximum (see Fig. 2(e)). We assume that the presence and the different diameters of the dark rings around the different clusters is caused by the different bonding of the clusters to the InAs substrate. This assumption is supported by the fact that different Co clusters look quite differently on STM images taken at the same value of the tunneling bias voltage. $I\left(V_{t}\right)$ curves also strongly differ when measured above different clusters. Curves, which are measured above clusters that are surrounded by a ring-like feature, reveal the presence of a narrow plateau (width of about $0.1 \mathrm{~V}$ ) around the Fermi level. On the other hand, clusters, which are not surrounded by a ring-like feature, reveal the presence of a wider plateau in the $I\left(V_{t}\right)$ curve (width is $0.5-0.7 \mathrm{~V}$ ). These findings clearly require further investigation.

The maximum observable ring radius is determined by the fact that the region with negative differential conductance (see Fig. 2(e)) becomes masked by the rapidly growing tail of the $I\left(V_{t}\right)$ characteristics at more negative bias voltages. For the Co clusters in the selected area this masking occurs for voltages $V_{t} \lesssim V_{t M A X} \simeq-0.7 \mathrm{~V}$ while for other areas the value of $V_{t M A X}$ will be different. In this bias voltage range the behavior of the tunneling conductance becomes much more complicated. As can be seen in Fig. 2(b), ring-like protrusions are observed in the LDOS image rather than ring-like depressions. Some of the rings become partly dark and partly bright. For these more negative bias voltages, the exact relation between the local slope of the $I\left(V_{t}\right)$ curve and the depression of the tunneling current becomes important. More subtle details, such as the presence of neighboring clusters, impurity atoms or surface defects, will then have a pronounced influence on the imaging of the clusters.

The large grid size that has been used for acquiring the CITS data allows us to present the data in another inter- 

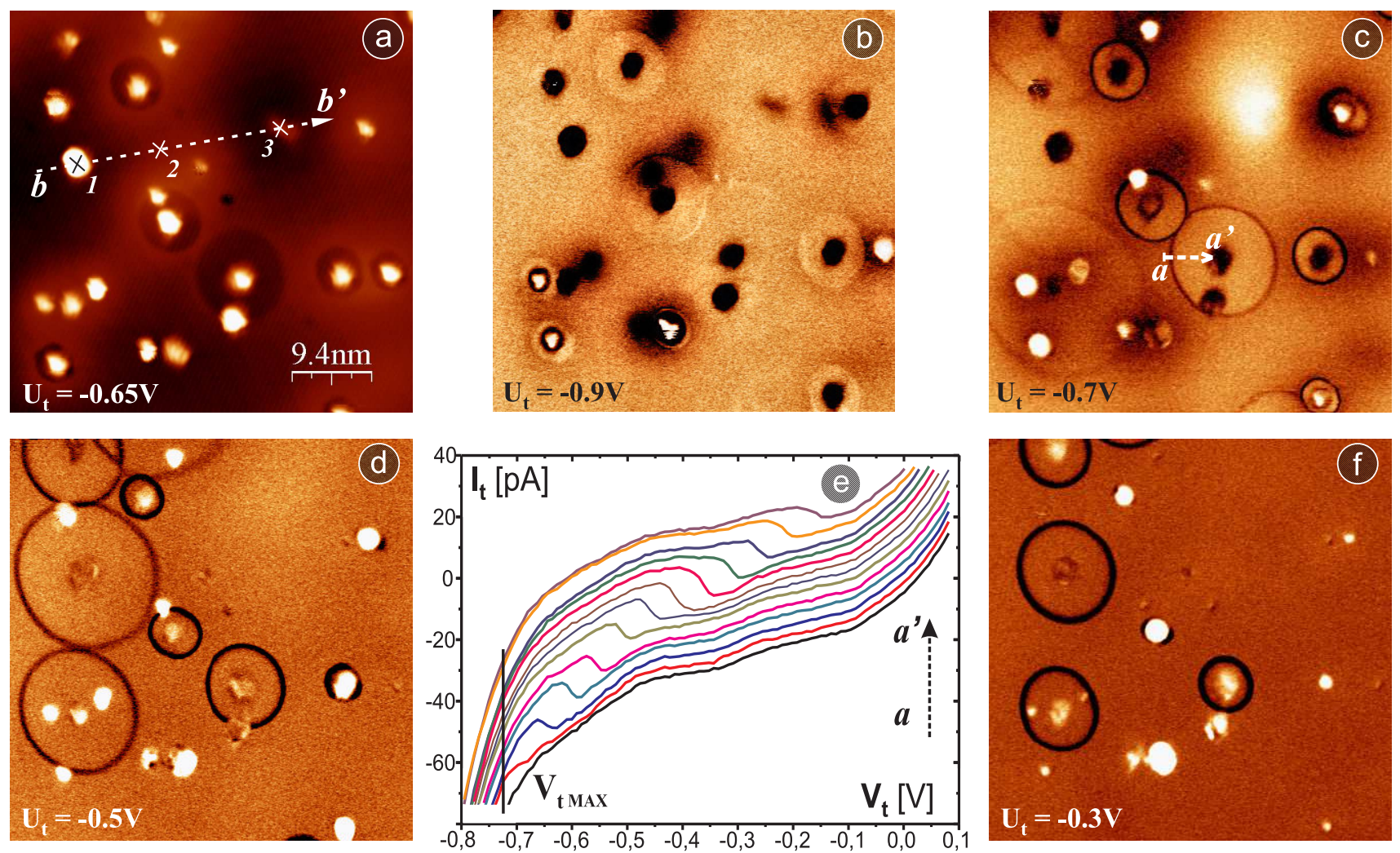

FIG. 2: (a) Experimental constant current topographical STM image of the Co-InAs(110) surface with a Co coverage of about $0.02 \mathrm{ML}$ obtained at $V_{t}=-0.65 \mathrm{~V}$ and $I=400 \mathrm{pA}$. The image size is $47 \times 47 \mathrm{~nm}^{2}$. (b), (c), (d) and (f) are maps of the measured differential conductance $d I / d V_{t}\left(x, y, V_{t}\right)$ for the same surface region as in (a) at different tunneling bias voltage. The tunneling current setpoint is $I_{t}=150 \mathrm{pA}$, while the tunneling bias voltage is indicated at the bottom of the maps. Ring-like features, whose appearance is strongly affected by the applied negative bias voltage, can be observed. (e) Set of $I\left(V_{t}\right)$ curves extracted from the CITS data (see text) along the line $a a^{\prime}$ (see (c)). The different curves are offset for clarity. Regions with negative differential conductance are clearly visible. The value of the tunneling bias voltage corresponding to the maximum observable ring radius (see text) is marked as $V_{t M A X}$. The ring in (c) that is below the line $a a^{\prime}$ has almost reached its maximum radius.

esting way. Figure 3(a) gives a 2D map of the variation of the normalized tunneling conductance data along the cross-section $b b^{\prime}$ in Fig. 2(a). The horizontal axis corresponds to the spatial coordinate, while the vertical axis corresponds to the tunneling bias voltage, and the color intensity corresponds to the measured value of the normalized tunneling conductance (LDOS). In Fig. 3(b) we show $\left(d I / d V_{t}\right) /\left(I / V_{t}\right)$ at the three points that are marked by the crosses on the cross-section $b b^{\prime}$ in Fig. 2(a). These three points (1, 2, and 3) are marked in Fig. 3(a) as well.

From Fig. 3(a) it is clear that a Co cluster affects the LDOS in the conduction and valence bands only very locally. Except for a limited voltage range near $V_{t}=0$, the influence of a cluster on the LDOS is very rapidly decaying outside the cluster. Inside the cluster, the conductivity in the valence band is considerably suppressed. Some of the energy levels of the Co cluster become visible in the voltage range corresponding to the band gap and the conduction band. In Fig. 3(a) there are two tilted dark lines that approximately start from the peak in the normalized tunneling conductance of the cluster and that extend over a distance of about $8 \mathrm{~nm}$ into the defect free surface area. These two dark lines directly reflect the presence of the dark rings around the clusters as well as the dependence of the ring diameter on the tunneling bias voltage, which is clearly non-linear.

The two peaks in the normalized tunneling conductance at $-0.3 \mathrm{~V}$ and at $-0.45 \mathrm{~V}$ (see curve (a) in the inset to Fig. (1) also appear in Fig. 3(a) in the defect free surface area. The peaks are visible as perfectly straight, horizontal stripes. This implies that there occurs no noticeable change in the "rigid" (both bands move together) band shifts relative to the original bands of the p-type InAs. This is rather surprising, because a charged cluster and a charged impurity atom, separated by a distance of about $20 \mathrm{~nm}$, are surrounding the scanned area, and one might therefore expect changes to occur in the measured band shift. The absence of changes in the rigid band shift may be accounted for in the following way. First, both the Co cluster and the Mn impurity atom can be treated as point charges. It is known that a point charge potential does not strongly affect the band bending, but rather gives rise to fine structure in the LDOS 12]. Second, the variations of the LDOS around the Co cluster 
are strongly confined in space, while the Mn impurity atom alters the LDOS mostly near the top of the valence band. Hence, the conduction band remains almost undisturbed, with the conduction band bottom $E_{C}$ appearing as a straight line in Fig. 3(a). When the tunneling bias voltage $V_{t}$, which is sweeping during the measurements of the tunneling conductance, reaches the band gap region and goes down to the top of the valence band, the energy levels $E_{0}, E_{1}$ of the 2D quantum well become populated. Taking into account the very high electron mobility along the surface [16], we may assume that in this bias range perfect screening by the $2 \mathrm{D}$ electron gas is taking place, hiding other details in the electronic structure.

On the other hand, when the $2 \mathrm{D}$ subband $E_{1}$ is shifted below the Fermi level, the tunneling bias dependent band bending noticeably slows down. This can be accounted for by the overlapping of the higher 2D subbands, which form a relatively wide continuum of states, thus pinning the Fermi level in this continuum. In this case the surface band structure "stabilizes", i.e., an increase of the absolute value of the negative bias voltage leads only to slight changes in 2D subband energy.

Another important observation related to Fig. 3(a) is the influence of the Mn impurity atoms on (below) the Co-InAs(110) surface on the tunneling spectra. In the vicinity of a $\mathrm{Mn}$ atom the LDOS is affected deep inside the conduction and valence bands (not visible in Fig. 3(a)). Additionally, an intense peak appears in Fig. 3(a) near the valence band top and most probably this peak reflects the energy position of the Mn acceptor band. We note that the spatial extent of this peak is relatively large, about $7 \mathrm{~nm}$.

\section{DISCUSSION}

In order to explain the observed effects consistently one needs to take into account the presence of nonequilibrium and interference effects in the small tunneling junction [17], which can be described using the Keldysh diagram technique.

We need to explain two main peculiarities that are related to the observation of the ring-like features. First, the presence of the NDC region in the tunneling conductivity curves has to be accounted for. Second, the limited spatial extent of the circular features requires an explanation. Accordingly, this discussion section has been divided into two subsections.

\section{A. Negative differential conductance}

We start from the following model Hamiltonian $\hat{H}$ :

$$
\hat{H}=\hat{H}_{0}+\hat{H}_{t u n}+\hat{H}_{c l}+\hat{H}_{Q W},
$$
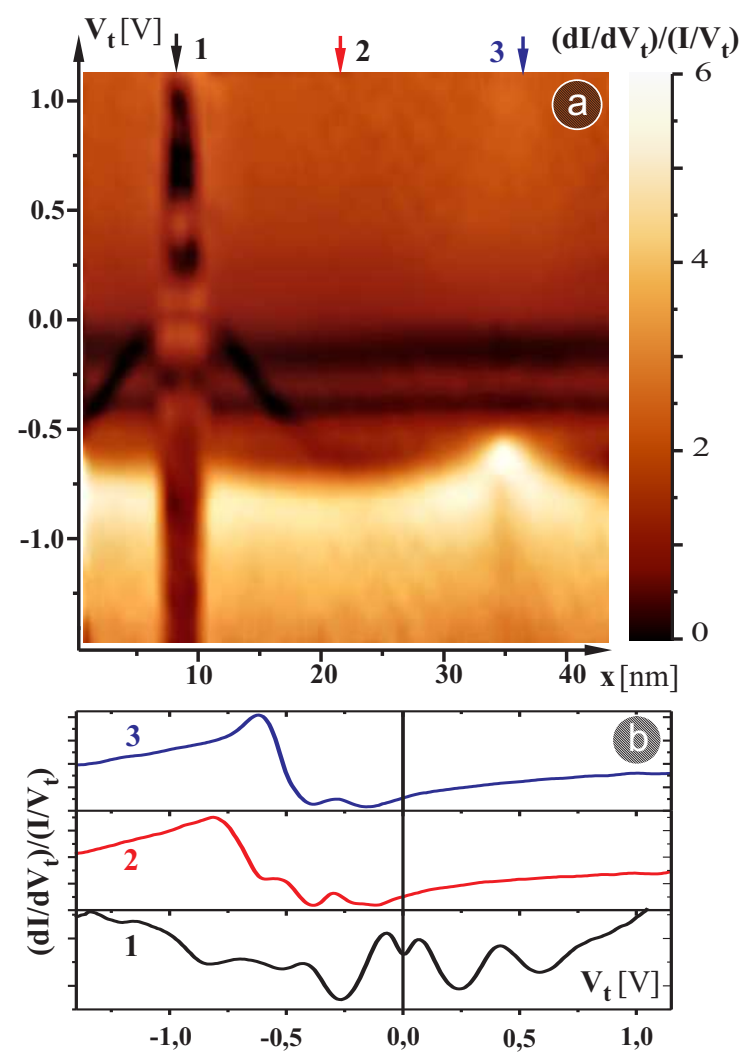

FIG. 3: (a) 2D representation of the normalized differential tunneling conductance, extracted from the CITS data (see text) along the line $b b^{\prime}$ indicated in Fig. 2(a). The horizontal axis corresponds to the spatial coordinate, while the vertical axis corresponds to the tunneling bias voltage. The color contrast in the image corresponds to the value of the differential tunneling conductance as given by the color scale on the right hand side. (b) Three normalized differential conductance curves measured at the three points $(1,2$, and 3 , see (a)) that are marked by the crosses along the line $b b^{\prime}$ in Fig. 2(a). The curves are measured above a cluster (1), above a defect free surface area (2) and above a Mn impurity atom (3).

where

$$
\hat{H}_{0}=\sum_{\mathbf{q}, \sigma}\left(\varepsilon_{q}-\mu\right) c_{\mathbf{q} \sigma}^{+} c_{\mathbf{q} \sigma}+\sum_{\mathbf{p}, \sigma}\left(\varepsilon_{p}-\mu-e V\right) c_{\mathbf{p} \sigma}^{+} c_{\mathbf{p} \sigma}
$$

describes the non-interacting electrons in the two electrodes: tip states $(\mathbf{p} \sigma)$, and bulk states $(\mathbf{q} \sigma)$. The part $\hat{H}_{c l}$ corresponds to the electron states in the cluster:

$$
\hat{H}_{c l}=\varepsilon_{a} \sum_{\sigma} a_{\sigma}^{+} a_{\sigma},
$$

where the operator $a_{\sigma}\left(a_{\sigma}^{+}\right)$destroys (creates) an electron in the cluster with spin $\sigma$. The part $\hat{H}_{Q W}$ describes the electron states in the quantum well resulting from the Co cluster deposition and the STM tip induced band bending:

$$
\hat{H}_{Q W}=\sum_{\mathbf{k}, m, \sigma} \varepsilon_{\mathbf{k} m \sigma} c_{\mathbf{k} m \sigma} c_{\mathbf{k} m \sigma}^{+},
$$




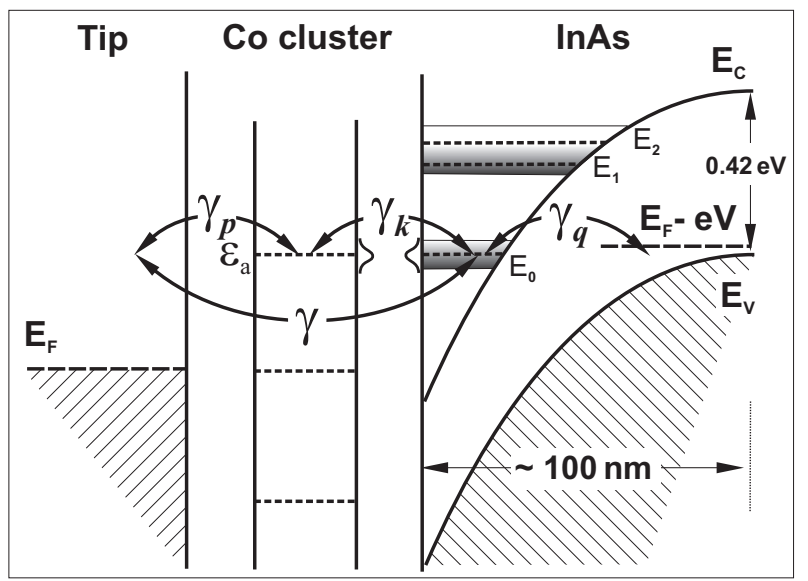

FIG. 4: Schematic representation of the tunneling processes occurring in the system consisting of the STM tip, a Co cluster and the $\operatorname{InAs}(110)$ substrate. $\varepsilon_{a}$ is the Co cluster energy level that is participating in the tunneling. $E_{0}$ is the first $2 \mathrm{D}$ subband, which has a non-zero but narrow width, as indicated by the gray stripe. A gray stripe is also used to mark the presence of the higher $2 \mathrm{D}$ subbands, which form a continuum of states due to their broadening. The system is shown at non-zero bias voltage when $\varepsilon_{a}$ and $E_{0}$ are aligned and a dark ring appears around the Co cluster in the maps of the differential tunneling conductance.

where $c_{\mathbf{k} m \sigma}\left(c_{\mathbf{k} m \sigma}^{+}\right)$destroys (creates) an electron with spin $\sigma$ in the quantum well, $m$ is the number labeling the $2 \mathrm{D}$ bands and $\mathbf{k}$ is the $2 \mathrm{D}$ wavevector. $\hat{H}_{\text {tun }}$ is responsible for tunneling transitions from the quantum well and from the cluster into each of the electrodes (tip or substrate):

$$
\begin{array}{r}
\hat{H}_{t u n}=\sum_{\mathbf{p}, \sigma} T_{\mathbf{p} \sigma} c_{\mathbf{p} \sigma}^{+} a_{\sigma}+\sum_{\mathbf{k}, m, \sigma} T_{\mathbf{k} m} c_{\mathbf{k} m \sigma}^{+} a_{\sigma}+ \\
\sum_{\mathbf{p}, \mathbf{k}, m, \sigma} W e^{i \varphi(r)} c_{\mathbf{k} m \sigma}^{+} c_{\mathbf{p} \sigma}+\sum_{\mathbf{q}, \mathbf{k}, m, \sigma} T_{\mathbf{q}} c_{\mathbf{k} m \sigma}^{+} c_{\mathbf{q} \sigma}+h . c .
\end{array}
$$

$T_{\mathbf{p} \sigma}, T_{\mathbf{k} m}, T_{\mathbf{q}}$ and $W$ are the relevant tunneling matrix elements: $T_{\mathbf{k} m}$ for tunneling from the cluster level to the quantum well subbands, $T_{\mathbf{p} \sigma}$ for tunneling from the cluster level to the STM tip continuum, $T_{\mathbf{q}}$ for tunneling from the quantum well level to the bulk of the semiconductor, $W$ for tunneling from the quantum well level to the STM tip.

The differential tunneling conductance can then be written as [18, 19]

$$
\begin{array}{r}
\frac{d I}{d V} \propto \Gamma \nu_{k}+\frac{2 \sqrt{\gamma_{p} \gamma_{k} \Gamma \nu_{k}\left(1-\Gamma \nu_{k}\right)^{2}}}{\left(1+\Gamma \nu_{k}\right)^{2}} \cos \varphi \operatorname{Re} G^{R}(e V) \\
-\frac{2 \gamma_{p} \gamma_{k}\left(\left(1+\Gamma \nu_{k}\right)^{2}-4 \Gamma \nu_{k} \cos ^{2} \varphi\right)}{\left(\gamma_{p}+\gamma_{k}\right)\left(1+\Gamma \nu_{k}\right)^{3}} \operatorname{Im} G^{R}(e V) \\
+\frac{2 \Gamma \nu_{k}\left(\gamma_{k}+\gamma_{p}\right)}{\left(1+\Gamma \nu_{k}\right)^{3}} \operatorname{Im} G^{R}(e V)
\end{array}
$$

were $\nu_{k}, \nu_{p}$ and $\nu_{q}$ are the density of states in the quantum well, the STM tip and the bulk semiconductor, re- spectively. The cluster density of states is proportional to $\operatorname{Im} G^{R}(\omega)$.

The different tunneling rates in Eq. (11) are defined as follows (see Fig. (4): $\gamma_{k}=T_{k m}^{2} \nu_{k m}$ is the tunneling transition rate from a cluster level to the quantum well subbands, $\gamma_{p}=T_{p}^{2} \nu_{p}$ is the tunneling transition rate from a cluster level to the STM tip, $\gamma_{q}=T_{q}^{2} \nu_{q}$ is the tunneling transition rate from the quantum well to the bulk of the semiconductor, and $\gamma=W^{2} \nu_{p}$ is the tunneling transition rate from the quantum well to the STM tip. Finally, $\Gamma$ is defined as $\Gamma=\gamma \gamma_{q} /\left(\gamma+\gamma_{q}\right)$. We omit the subband index $m$, because only the lowest subband plays the most important role in our model.

As stated above, a Co cluster acts as a donor, and consequently it is positively charged. In case of nonequilibrium, the energy of a cluster level $\varepsilon_{a}$, which is participating in the tunneling (see Fig. (4), depends on the electron filling number $\left\langle n_{a}\right\rangle$ of this level:

$$
\varepsilon_{a}=\varepsilon_{0}+U\left(n_{0}-<n_{a}>\right),
$$

where $\varepsilon_{0}$ is the unperturbed position of the cluster energy level, $n_{0}$ is the equilibrium cluster level filling number, and $U$ is the Coulomb interaction energy of the localized charges 17.

For STM junctions we can assume that $\Gamma \nu_{k}<<1$, because $\Gamma$ is of order of tunneling rates and $\nu_{k} \sim 1 / D$, where $D$ is the width of conduction (valence) band in InAs. Equation (1) then reduces to

$$
\begin{array}{r}
\Delta\left(\frac{d I}{d V}\right) \propto 2\left|\operatorname{Im} G^{R}(\omega)\right| \frac{\gamma_{p} \gamma_{k}-\Gamma \nu_{k}\left(\gamma_{p}+\gamma_{k}\right)^{2}}{\gamma_{p}+\gamma_{k}}+ \\
2 \operatorname{Re} G^{R}(\omega) \sqrt{\gamma_{p} \gamma_{k} \Gamma \nu_{k}} \cos \varphi
\end{array}
$$

where $\Delta$ refers the the extra contribution to the tunneling conductance resulting from the non-equilibrium effects.

Here $\varphi$ is the pase angle, ascuired due to difference of wavefunctions of direct (STM tip-2D subband) and indirect resonant tunneling. The complicated coefficient in front of $\operatorname{Im} G^{R}(\omega)$ and the presence of $\operatorname{Re} G^{R}(\omega)$ in Eq. (2) also results from interference between direct tuneling from the STM tip to a quantum well subband, and indirect resonant tunneling via the cluster level. Consequently, the involved tunneling rates determine the sign and the magnitude of the additional current resulting from the non-equilibrium and interference processes.

For each tip position the value of $\gamma_{p}$ is fixed, while $\gamma_{k}$ can be varied by changing the applied bias voltage. $\gamma_{k}$ depends on the relative position of the cluster level $\varepsilon_{a}$ and of the first $2 \mathrm{D}$ subband $E_{0}$. Both energies are bias dependent: $\varepsilon_{a}$ trough $\left\langle n_{a}\right\rangle$ and $E_{0}$ through the band bending. It is important to note that the $2 \mathrm{D}$ subband $E_{0}$ is relatively narrow. Electrons can be injected into this subband either from the STM tip $(\gamma)$ or from the Co cluster $\left(\gamma_{k}\right)$. In both cases the wavevector component parallel to the surface, $k_{\|}$, is small. In close analogy with angle resolved photoemission spectroscopy experiments [20], only a narrow range of energies is available 
for tunneling into the $2 \mathrm{D}$ subband $E_{0}$ (see Fig. (4). In general, the $k_{\| \mid}$component needs to be taken into account in STM experiments 21].

We assume that the unperturbed Co cluster energy level $\varepsilon_{0}$ (at zero bias voltage) is positioned close to $E_{F}$ below the first $2 \mathrm{D}$ subband $E_{0}$, i.e, the two energy levels are not aligned. This assumption is strongly supported by the experimental STS data (see Fig. 3 for details).

We now proceed with a more detailed analysis of the bias voltage dependence of $\Delta(d I / d V)$. The first term in Eq. (2), which varies proportional to the cluster density of states, can change its sign. If $\gamma_{p} \gamma_{k}<<\Gamma \nu_{k}\left(\gamma_{p}+\gamma_{k}\right)^{2}$ (let us assume $\gamma_{p}<<\gamma_{k}$ ) the sign of the coefficient that appears in front of $\operatorname{Im} G^{R}(\omega)$ is negative. For the assumed conditions, the coefficient that appears in front of $\operatorname{Re} G^{R}(\omega)$ is much smaller than the coefficient next to $\operatorname{Im} G^{R}(\omega)$. The tunneling conductance curve is an inverted Lorentzian-shaped curve that is slightly asymmetric because of the $\operatorname{Re} G^{R}(\omega)$ contribution. The position of the dip in the tunneling conductance curve can be connected to the $\varepsilon_{a}\left(\left\langle n_{a}\right\rangle\right)$ dependence. The closer to the cluster the STM tip is, the higher is the value of $\gamma_{p}$ and the lower is the value of the non-equilibrium filling $\left.<n_{a}\right\rangle$. The cluster energy level $\varepsilon_{a}$ is pushed higher, and the dip in the tunneling conductance curve can be observed at smaller absolute values of the applied bias voltage (see Fig (2). The dark rings that appear in the STS images shrink when the absolute value of the negative tunneling bias decreases within a cluster dependent range of negative voltages.

\section{B. Spatial extent of ring-like features}

The most probable physical mechanism causing the formation of narrow (of the order of the interatomic distance) ring-like features in real space is some kind of resonant tunneling. When the STM tip changes its spatial position, localized energy levels that participate in the tunneling processes are coming out of resonance or even are falling outside the tunneling window $\left[E_{F}, E_{F}-e V_{t}\right]$. We find that the simple cluster charging model used in [15, 22] is not adequate for describing our Co-InAs system. This is mainly due to the fact that on-site Coulomb repulsion for the cluster is the strongest bias dependent effect. This leads to variations of the cluster level filling number, and consequently to changes in the cluster level energy. Starting from this finding we are able to analyze in the framework of our model how spatially narrow circular features can be formed around the Co clusters.

For each value of the tunneling bias voltage $V_{t}$ the value of $\gamma_{p}$ can be altered by changing the STM tip to cluster distance. Consequently, $\left\langle n_{a}\right\rangle$, which depends on the ratio $\gamma_{k} / \gamma_{p}$ also changes, giving rise to a shift of $\varepsilon_{a}\left(\left\langle n_{a}\right\rangle\right)$ and to variations of $\gamma_{k}$. When moving the STM tip farther away from the cluster, $\gamma_{p}$ is decreasing, leading to an increase of $\left\langle n_{a}\right\rangle$ and to a lowering of $\varepsilon_{a}$. At a certain distance $d_{\max }$, the cluster energy level $\varepsilon_{a}\left(<n_{a}>\right)$ starts to becoming out of resonance with the narrow $2 \mathrm{D}$ subband $E_{0}$. Since there is now an alignment with the tail of the broadened $E_{0}$ level, the value of the tunneling current drops. At the same time, the value of $\gamma_{k}$ decreases, resulting in a decrease of the nonequilibrium occupation $\left\langle n_{a}\right\rangle$, and consequently $\varepsilon_{a}$ is pushed higher. The cluster level $\varepsilon_{a}$ then jumps to its equilibrium position $\varepsilon_{0}$. We assume that at the STM tip to cluster distance $d_{\max }$ the tunneling rate $\gamma_{p}$, which exponentially decreases with increasing distance, becomes negligible $\left(\gamma_{p} \approx 0\right)$. The STM tunneling system does no longer feel the STM tip - cluster interaction, and the value of the tunneling current returns to its "normal" value. In other words, when at a certain tunneling bias voltage in the range where a dark ring appears in the LDOS around the Co cluster, the STM tip to cluster distance $d$ reaches the value $d_{\max }$, some kind of positive feedback, caused by mutually coupled variations of $\gamma_{p}, \gamma_{k}$ and $\left\langle n_{a}\right\rangle$, switches on. This positive feedback abruptly shifts the cluster energy level out of resonance with the $2 \mathrm{D}$ subband. This can account for the very small spatial width of the rings that appear in the LDOS as well as for the non-linear dependence of the ring diameter on the STM tip to cluster distance (see Fig. 3).

We note that for specific tunneling conditions the first term in Eq. (2) can become positive, causing the observation of bright rings or protrusions in the images of the differential conductance. A dark ring can turn into a bright one at high values of the tunneling bias voltage, as can be seen in Fig. 2(b).

Our theoretical model does not include any assumptions concerning the physical origin of the localized states through which resonant tunneling can occur. Therefore, this model should also be applicable to the case of an individual impurity atom near the surface. Ring-like features around individual impurities were indeed observed in our experiments (to be published) as well as on the GaAs(110) surface [22].

\section{CONCLUSION}

In conclusion, relying on combined STM and STS measurements at low temperature, we have been able to identify the presence of ring-like features around Co metal clusters on a p-type $\operatorname{InAs}(110)$ surface that is prepared by in situ cleavage of a single crystal in UHV. The ringlike features become clearly visible in the maps of the differential normalized conductance for a certain range of negative tunneling bias voltages, due to the presence of a region with negative differential conductance in the $I\left(V_{t}\right)$ dependence. The diameter of the rings is decreasing when decreasing the absolute value of the tunneling bias voltage. The possibility to observe the ring-like features around a Co cluster depends on the bonding of the cluster to the InAs(110) surface.

A theoretical model was developed, which takes into account the non-equilibrium effects that occur in the 
small STM tunneling junction. The model we propose allows us to explain the main experimental findings. In the framework of the model the appearance of the ringlike features structures is accounted for by the interference of direct (between the STM tip and the QW) and indirect tunneling via a Co cluster.

\section{Acknowledgments}

This research in Moscow has been supported by the RFBR grants 05-02-19806-MF-a and 06-02-17076-a. The research in Leuven has been supported by the Fund for Scientific Research - Flanders (Belgium) as well as the Research Fund of the K.U.Leuven. We thanks A. A. Ezhov for the technical support. The authors also thank the WSxM team (www.nanotec.es).
[1] D. C. Tsui, Phys. Rev. Lett. 24, 303 (1970).

[2] M. G. Betti, V. Corradini, G. Bertoni, P. Casarini, C. Mariani, and A. Abramo, Phys. Rev. B 63, 155315 (2001).

[3] M. Morgenstern, J. Wiebe, A. Wachowiak, M. Getzlaff, J. Klijn, L. Plucinski, R. L. Johnson, and R. Wiesendanger, Phys. Rev. B 65, 155325 (2002).

[4] S. Abe, T. Inaoka, and M. Hasegawa, Phys. Rev. B 66, 205309 (2002).

[5] www.omicron.de.

[6] R. Wiesendanger, Scanning Probe Microscopy and Spectroscopy (Cambridge University Press, 1994).

[7] H. Carstensen, R. Claessen, R. Manzke, and M. Skibowski, Phys. Rev. B 41, 9880 (1990).

[8] C. B. M. Andersson, J. N. Andersen, P. E. S. Persson, and U. O. Karlsson, Surf. Sci. 398, 395 (1998).

[9] L. Ö. Olsson, C. B. M. Andersson, M. C. Håkansson, J. Kanski, L. Ilver, and U. O. Karlsson, Phys. Rev. Lett. 76, 3626 (1996).

[10] H. S. Karlsson, R. Viselga, and U. O. Karlsson, Surf. Sci. 402, 590 (1998).

[11] P. I. Arseev, N. S. Maslova, V. I. Panov, S. V. Savinov, and C. Van Haesendock, JETP Lett. 77, 172 (2003).

[12] N. Maslova, Habilitation Thesis (Moscow State Univer- sity, 2002).

[13] I. Vurgaftmana, J. R. Meyer, and L. R. Ram-Mohan, Appl. Phys. Rev. 89, 5815 (2001).

[14] R. M. Feenstra, G. Meyer, F. Moresco, and K. H. Rieder, Phys. Rev. B 66, 165204 (2002).

[15] J. W. G. Wildöer, A. J. A. van Roij, C. J. P. M. Harmans, and H. van Kempen, Phys. Rev. B 53, 10695 (1996).

[16] T. I. Voronina, T. S. Lagunova, M. P. Mikhailova, K. D. Moiseev, A. F. Lipaev, and Y. P. Yakovlev, Semiconductors 40, 497 (2006).

[17] P. I. Arseev, N. S. Maslova, and S. V. Savinov, JETP Lett. 68, 320 (1998).

[18] W. Hofstetter, J. König, and H. Schoeller, Phys. Rev. Lett. 87, 156803 (2001).

[19] P. I. Arseev, N. S. Maslova, V. I. Panov, and S. V. Savinov, JETP 94, 191 (2002).

[20] V. Y. Aristov, G. Le Lay, V. M. Zhilin, G. Indlekofer, C. Grupp, A. Taleb-Ibrahimi, and P. Soukiassian, Phys. Rev. B 60, 7752 (1999).

[21] N. D. Jäger, E. R. Weber, K. Urban, and P. Ebert, Phys. Rev. B 67, 165327 (2003).

[22] P. Koenraad, private communication. 